\title{
FIGO Stage IIIA1
}

National Cancer Institute

\section{Source}

National Cancer Institute. FlGO Stage IIIA1. NCI Thesaurus. Code C128102.

A FIGO stage term that applies to ovarian cancer, fallopian tube cancer, and primary peritoneal cancer. It refers to cancer with positive retroperitoneal lymph nodes only. 